PROCEEDINGS OF THE

AMERICAN MATHEMATICAL SOCIETY

Volume 138, Number 4, April 2010, Pages 1305-1315

S 0002-9939(09)10150-8

Article electronically published on November 25, 2009

\title{
INEQUALITIES OF CHERNOFF TYPE FOR FINITE AND INFINITE SEQUENCES OF CLASSICAL ORTHOGONAL POLYNOMIALS
}

\author{
RYSZARD SMARZEWSKI AND PRZEMYSŁAW RUTKA \\ (Communicated by Peter A. Clarkson)
}

\begin{abstract}
In this paper we present two-sided estimates of Chernoff type for the weighted $L_{w}^{2}$-distance of a smooth function to the $k$-dimensional space of all polynomials of degree less than $k$, whenever the weight function $w$ solves the Pearson differential equation and generates a finite or infinite sequence of classical orthogonal polynomials. These inequalities are simple corollaries of a unified general theorem, which is the main result of the paper.
\end{abstract}

\section{INTRODUCTION AND PRELIMINARIES}

Let a weight function $w$ be positive on a finite or infinite interval $(a, b)$, normalized by the condition

$$
\int_{a}^{b} w(x) d x=1
$$

Moreover, let $L_{w}^{2}(a, b)$ denote the real Hilbert function space with the inner product

$$
(f, g)_{w}=\int_{a}^{b} f(x) g(x) w(x) d x
$$

and the norm $\|f\|_{w}=\sqrt{(f, f)_{w}}$. Suppose that all polynomials belong to $L_{w}^{2}(a, b)$ and define the minimal $L_{w}^{2}$-distance

$$
E_{w, k}(f)=\inf _{p \in \mathcal{P}_{k-1}}\|f-p\|_{w}, f \in L_{w}^{2, k}(a, b),
$$

of $f$ to the $k$-dimensional space $\mathcal{P}_{k-1}$ of all polynomials of degree less than $k$, where $L_{w}^{2, k}(a, b)$ denotes the space of all functions $f$ in $L_{w}^{2}(a, b)$ such that the $(k-1)$ th derivative $D^{k-1} f$ is absolutely continuous and $D^{k} f$ belongs to $L_{w}^{2}(a, b)$.

Throughout the paper, we assume that the weight function $w$ is a solution of the Pearson differential equation

$$
D(A w)=B w
$$

with boundary conditions of the form

$$
\lim _{x \downarrow a} A(x) w(x)=\lim _{x \uparrow b} A(x) w(x)=0
$$

Received by the editors October 27, 2008, and, in revised form, June 17, 2009.

2000 Mathematics Subject Classification. Primary 26D10; Secondary 33C45, 60E15.

Key words and phrases. Chernoff-type inequalities, classical finite and infinite orthogonal polynomials, optimal constants, generic differential equations.

(C)2009 American Mathematical Society Reverts to public domain 28 years from publication 
with real polynomials

$$
A(x)=a_{0}+a_{1} x+a_{2} x^{2} \text { and } B(x)=b_{0}+b_{1} x
$$

such that $A(x)>0$ on $(a, b)$ and $b_{1} \neq 0$. Then the finite or infinite sequence of polynomials $Q_{n}\left(0 \leq n<n_{w}\right)$, orthogonal with respect to the inner product $(\cdot, \cdot)_{w}$, is said to be a finite or infinite sequence of classical orthogonal polynomials, and each $Q_{n}$ is in addition a solution of the following generic differential equation:

$$
D(A w D Q)=\lambda_{n} w Q
$$

with the coefficient $\lambda_{n}$ equal to

$$
\lambda_{n}=n\left[(n-1) a_{2}+b_{1}\right] ;
$$

cf. Bochner 4, Hahn [9, Krall [13, 14, 15], Agarwal et al. 11, Andrews et al. [3], Chihara 6], Nikiforov et al. [19] and Suetin [23. Without loss of generality, it will be assumed that $n_{w}$ is the greatest upper bound of all numbers $n$ such that polynomials $Q_{n}\left(0 \leq n<n_{w}\right)$ are classical orthogonal polynomials. In this case, the function $w$ will be called a classical weight function.

In view of Hahn 9] and Krall [13, 14, 15] (cf. also Agarwal et al. 11), the derivatives $D^{k} Q_{n}, n=k, k+1, \ldots$, of finite or infinite classical orthogonal polynomials $Q_{n}$ are also finite or infinite classical orthogonal polynomials. More precisely, these derivatives are orthogonal with respect to the inner product $(\cdot, \cdot)_{w_{k}}$ and each derivative $D^{k} Q_{n}$ satisfies the differential equation

$$
D\left(A w_{k} D Q\right)=\lambda_{n, k} w_{k} Q
$$

with the normalized weight function

$$
w_{k}=\frac{A^{k} w}{\gamma_{k}}, \gamma_{k}=\int_{a}^{b} A^{k}(x) w(x) d x,
$$

and

$$
\lambda_{n, k}=(n-k)\left[(n+k-1) a_{2}+b_{1}\right] .
$$

In this paper, we present a unified and simple proof of estimates of Chernoff type 22 for the class of all weighted distance functionals $E_{w, k}(f)$, with $w$ a classical weight function. For this purpose, we denote by

$$
\widehat{Q}_{k}(x)=d_{k} x^{k}+\cdots+d_{1} x+d_{0}
$$

the classical orthonormal polynomial $\widehat{Q}_{k}=Q_{k} /\left\|Q_{k}\right\|_{w}$ and note that the coefficient $d_{k}$ of the highest term $x^{k}$ in $\widehat{Q}_{k}(x)$ is always distinct from zero. It is also interesting to note that the number $D^{k} \widehat{Q}_{k}=k ! d_{k}$ plays a crucial role in the following main theorem of the paper. As we shall see in Section 2, this theorem gives a unified and simple approach to deal with all six classes of classical weight functions. In particular, it yields a simple new proof of Chernoff-type inequalities when $w$ is either the Hermite [5] or Jacobi [21, 22] classical weight function.

Theorem 1.1. Let $w$ be a normalized classical weight function defined by the Pearson differential equation. Then the inequalities

$$
\frac{1}{\left|D^{k} \widehat{Q}_{k}\right|}\left|\int_{a}^{b}\left(D^{k} f\right)(x) w_{k}(x) d x\right| \leq E_{w, k}(f) \leq \frac{1}{\left|D^{k} \widehat{Q}_{k}\right|}\left\|D^{k} f\right\|_{w_{k}}, 0 \leq k<n_{w},
$$


hold whenever $f$ belongs either to $L_{w_{k}}^{2, k}(a, b)$ or to $\mathcal{P}_{n_{w}-1}$ for $n_{w}=\infty$ or $n_{w}<$ $\infty$, respectively. Additionally, both these inequalities become equalities for every polynomial $f$ of degree $\leq k$.

Recall [22] that this two-sided inequality is analogous, in the case when $k=1$, to the two-sided inequality of the form

$$
\left[E G^{\prime}(X)\right]^{2} \leq \operatorname{Var}[G(X)] \leq E\left[G^{\prime}(X)\right]^{2},
$$

where $X$ is a normally distributed random variable with mean 0 and variance $1, G$ is an absolutely continuous function and $G(X)$ has finite variance. Moreover, both these inequalities become equalities if $G(X)$ is linear. Since the right hand side has been proved by Chernoff [5], it is often called a Chernoff inequality in probability theory.

In applications of Theorem 1.1 it is necessary to evaluate the constants $\gamma_{k}$ defined in (1.4). Therefore, it is convenient to compute the constant $\left|D^{k} \widehat{Q}_{k}\right|^{-1}$ from the formula presented in the next remark.

Remark 1.2. The constant $\left|D^{k} \widehat{Q}_{k}\right|^{-1}$ in the Chernoff-type inequalities can be evaluated from the formula

$$
\frac{1}{\left|D^{k} \widehat{Q}_{k}\right|}=\sqrt{\frac{\gamma_{k}}{k ! \prod_{i=0}^{k-1}\left[-(k+i-1) a_{2}-b_{1}\right]}}, 0<k<n_{w} .
$$

Moreover, it is equal to 1 in the case when $k=0$.

Remark 1.3. In order to compute the constant in the Chernoff-type inequalities, one can also apply the formula

$$
\frac{1}{\left|D^{k} \widehat{Q}_{k}\right|}=\frac{\left\|Q_{k}\right\|_{w}}{\left|D^{k} Q_{k}\right|}
$$

\section{Applications to the specific Classical Weight functions}

In this section we apply Theorem 1.1 together with Remark 1.2 to all six classes of classical weight functions, for which there exist six finite or infinite different sequences of classical orthogonal polynomials, up to a linear change of variable. More precisely, we will consider several weight functions $w_{k}(x), 0 \leq k<\infty$, and use the convention that $w(x)=w_{0}(x)$ on $(a, b)$. Note that all definitions and evaluations of integrals from this section are standard and can be found for example in $[2,3,18,10,11,12,18,23,24]$. Moreover, we note that the constants $\left|D^{k} \widehat{Q}_{k}\right|^{-1}$ from Theorem 1.1 will be computed below only in the nontrivial case when $k \geq 1$.

2.1. Hermite classical weight functions. Let $a=-\infty, b=+\infty, A(x)=1$, $B(x)=-2 x, w_{k}(x)=e^{-x^{2}} / \gamma_{k}$ and

$$
\gamma_{k}=\int_{-\infty}^{+\infty} e^{-x^{2}}=\sqrt{\pi}
$$


Then by applying Remark 1.2 we get

$$
\frac{1}{\left|D^{k} \widehat{Q}_{k}\right|}=\sqrt{\frac{\sqrt{\pi}}{k ! \prod_{i=0}^{k-1} 2}}=\sqrt{\frac{\sqrt{\pi}}{k ! 2^{k}}} .
$$

Hence Theorem 1.1 gives the inequalities

$$
\sqrt{\frac{\sqrt{\pi}}{k ! 2^{k}}}\left|\int_{-\infty}^{+\infty}\left(D^{k} f\right)(x) w_{k}(x) d x\right| \leq E_{w, k}(f) \leq \sqrt{\frac{\sqrt{\pi}}{k ! 2^{k}}}\left\|D^{k} f\right\|_{w_{k}}
$$

for all $f \in L_{w_{k}}^{2, k}(-\infty,+\infty)$ and $k=1,2, \ldots$, which were proved by Chernoff [5] in the case when $k=1$. Both inequalities are attained when $f$ is a polynomial of degree $\leq k$.

2.2. Jacobi classical weight functions. Let $(a, b)=(-1,1), \alpha>-1, \beta>-1$, $A(x)=1-x^{2}, B(x)=\beta-\alpha-(\alpha+\beta+2) x$ and

$$
w_{k}(x)=\frac{1}{\gamma_{k}}(1-x)^{\alpha+k}(1+x)^{\beta+k}, k=0,1, \ldots,
$$

where the constants $\gamma_{k}$ are equal to

$$
\begin{aligned}
\gamma_{k} & =\int_{-1}^{+1}(1-x)^{\alpha+k}(1+x)^{\beta+k} d x \\
& =2^{\alpha+\beta+2 k+1} \frac{\Gamma(\alpha+k+1) \Gamma(\beta+k+1)}{\Gamma(\alpha+\beta+2 k+2)} .
\end{aligned}
$$

Since $a_{2}=-1$ and $b_{1}=-(\alpha+\beta+2)$, it follows from Remark 1.2 that the constant $\left|D^{k} \widehat{Q}_{k}\right|^{-1}$ in Theorem 1.1 is given by the formula

$$
\begin{aligned}
\frac{1}{\left|D^{k} \widehat{Q}_{k}\right|} & =\sqrt{\frac{\gamma_{k}}{k ! \prod_{i=0}^{k-1}(\alpha+\beta+k+i+1)}} \\
& =\sqrt{\frac{2^{\alpha+\beta+2 k+1} \Gamma(\alpha+k+1) \Gamma(\beta+k+1)}{k !(\alpha+\beta+k+1)_{k} \Gamma(\alpha+\beta+2 k+2)}}
\end{aligned}
$$

where $(x)_{k}=x(x+1) \cdot \ldots \cdot(x+k-1)$ denotes the Pochhammer symbol. This result has been presented recently by Stepanov [21, 22] with a complicated long proof. Note that the simplest constants in the Chernoff-type inequalities are obtained for Legendre and Chebyshev weights. Indeed we have the following results.

( $i$ ) For the Legendre weight function $(\alpha=\beta=0)$, we have

$$
\gamma_{k}=2^{2 k+1} \frac{(k !)^{2}}{(2 k+1) !}, \frac{1}{\left|D^{k} \widehat{Q}_{k}\right|}=\frac{2^{k} k !}{(2 k) !} \sqrt{\frac{2}{2 k+1}} .
$$

(ii) In the case of the Chebyshev weight function of the first kind $\left(\alpha=\beta=-\frac{1}{2}\right)$,

$$
\gamma_{k}=\frac{\pi(2 k-1) ! !}{(2 k) ! !}, \frac{1}{\left|D^{k} \widehat{Q}_{k}\right|}=\frac{\sqrt{\pi / 2}}{k(2 k-2) ! !} .
$$


(iii) In the case of the Chebyshev weight function of the second kind $\left(\alpha=\beta=\frac{1}{2}\right)$,

$$
\gamma_{k}=\frac{\pi(2 k+1) ! !}{(2 k+2) ! !}, \frac{1}{\left|D^{k} \widehat{Q}_{k}\right|}=\frac{\sqrt{\pi / 2}}{(2 k) ! !}
$$

2.3. Laguerre classical weight functions. Let $a=0, b=+\infty, \alpha>-1, A(x)=$ $x, B(x)=1-\alpha-x$ and

$$
w_{k}(x)=\frac{1}{\gamma_{k}} x^{\alpha+k} e^{-x}
$$

with

$$
\gamma_{k}=\int_{0}^{+\infty} x^{\alpha+k} e^{-x} d x=\Gamma(\alpha+k+1) .
$$

Then it follows from Remark 1.2 that

$$
\frac{1}{\left|D^{k} \widehat{Q}_{k}\right|}=\sqrt{\frac{\Gamma(\alpha+k+1)}{k !}} .
$$

Thus Theorem 1.1 yields the Chernoff-type inequalities

$$
\sqrt{\frac{\Gamma(\alpha+k+1)}{k !}}\left|\int_{0}^{+\infty}\left(D^{k} f\right)(x) w_{k}(x) d x\right| \leq E_{w, k}(f) \leq \sqrt{\frac{\Gamma(\alpha+k+1)}{k !}}\left\|D^{k} f\right\|_{w_{k}}
$$

for all $f \in L_{w_{k}}^{2, k}(0,+\infty)$, which are optimal in the sense that they become equalities for polynomials of degree $\leq k$.

2.4. The generalized Bessel weight functions. Suppose that $a=0, b=+\infty$, $A(x)=x^{2}, B(x)=\alpha x+\beta, \alpha \notin\{0,-1,-2, \ldots\}$ and $\beta \neq 0$. Next define the normalized weight functions by

$$
w_{k}(x)=\frac{1}{\gamma_{k}} x^{\alpha+2 k-2} e^{-\frac{\beta}{x}},
$$

where

$$
\gamma_{k}=\int_{0}^{+\infty} x^{\alpha+2 k-2} e^{-\frac{\beta}{x}} d x=\beta^{\alpha+2 k-1} \Gamma(1-\alpha-2 k) .
$$

In this case the classical orthogonal polynomials

$$
B_{n}^{(\alpha, \beta)}(x)=\frac{c_{n}}{w(x)} D^{n}\left(w(x) x^{2 n}\right), w=w_{0},
$$

called the generalized Bessel polynomials [2, 17, 20, exist only for

$$
n<n_{w}=\left\lfloor\frac{1-\alpha}{2}\right\rfloor,
$$

where $\lfloor x\rfloor$ is the floor function. Hence one can apply Remark 1.2 to get

$$
\begin{aligned}
\delta_{k} & =\frac{1}{\left|D^{k} \widehat{Q}_{k}\right|}=\sqrt{\frac{\gamma_{k}}{k ! \prod_{i=0}^{k-1}[-(k+i-1)-\alpha]}} \\
& =\sqrt{\frac{\beta^{\alpha+2 k-1} \Gamma(1-\alpha-2 k)}{k !(2-\alpha-2 k)_{k}}} .
\end{aligned}
$$


Thus Theorem 1.1 yields the Chernoff-type inequalities

$$
\delta_{k}\left|\int_{a}^{b}\left(D^{k} p\right)(x) w_{k}(x) d x\right| \leq E_{w, k}(p) \leq \delta_{k}\left\|D^{k} p\right\|_{w_{k}}
$$

for all polynomials $p \in \mathcal{P}_{n_{w}-1}$ and $0<k<n_{w}$, which are attained for a polynomial $p$ of degree $\leq k$.

2.5. Jacobi weight functions on $[0, \infty)$. Let $a=0, b=+\infty, A(x)=x^{2}+x$, $B(x)=(2-\alpha) x+\beta+1, \beta>-1$. Moreover let the normalized weight function be defined by

$$
w_{k}(x)=\frac{x^{\beta+k}}{\gamma_{k}(1+x)^{\alpha+\beta-k}}, k=0,1, \ldots,
$$

where $\gamma_{k}$ is equal to

$$
\begin{aligned}
\gamma_{k} & =\int_{0}^{+\infty} \frac{x^{\beta+k}}{(1+x)^{\alpha+\beta-k}} d x \\
& =\frac{\Gamma(\alpha-2 k-1) \Gamma(\beta+k+1)}{\Gamma(\alpha+\beta-k)} .
\end{aligned}
$$

In this case there exists [12, 17, 20] only a finite sequence of classical orthogonal polynomials

$$
M_{n}^{(\alpha, \beta)}(x)=\frac{c_{n}}{w(x)} D^{n}\left(w(x)\left(x^{2}+x\right)^{n}\right), 0 \leq n<n_{w}, w=w_{0},
$$

where

$$
n_{w}=\left\lfloor\frac{\alpha-1}{2}\right\rfloor .
$$

Since $a_{2}=1$ and $b_{1}=2-\alpha$, it follows from Theorem 1.1 and Remark 1.2 that the Chernoff-type inequalities (2.1) hold with the constant

$$
\delta_{k}=\sqrt{\frac{\Gamma(\alpha-2 k-1) \Gamma(\beta+k+1)}{k !(\alpha-2 k)_{k} \Gamma(\alpha+\beta-k)}} .
$$

They become identities for every polynomial of degree $\leq k$.

2.6. Pseudo-Jacobi weight functions. Let $a=-\infty, b=+\infty$ and

$$
\begin{aligned}
& a_{2}=1, a_{1}=2(A B+C D) /\left(A^{2}+C^{2}\right), a_{0}=\left(B^{2}+D^{2}\right) /\left(A^{2}+C^{2}\right), \\
& b_{1}=2(1-\alpha), b_{0}=\beta(A D-B C) /\left(A^{2}+C^{2}\right)+(1-\alpha) a_{1}
\end{aligned}
$$

with $A D-B C>0$ and $A^{2}+C^{2}>0$. Following [17, 18, 20] we define the normalized weight functions by

$$
w_{k}(x)=\frac{1}{\gamma_{k}}\left(A^{2}+C^{2}\right)^{\alpha-k}\left[(A x+B)^{2}+(C x+D)^{2}\right]^{-\alpha+k} e^{\beta \arctan \left(\frac{A x+B}{C x+D}\right),}
$$

where

$$
\begin{aligned}
\gamma_{k} & =\left(A^{2}+C^{2}\right)^{\alpha-k} \int_{-\infty}^{+\infty}\left[(A x+B)^{2}+(C x+D)^{2}\right]^{-\alpha+k} e^{\beta \arctan \left(\frac{A x+B}{C x+D}\right)} d x \\
& =\frac{\left(A^{2}+C^{2}\right)^{\alpha-k}}{(A D-B C)^{2 \alpha-2 k-1}} \int_{-\pi / 2}^{\pi / 2}(A \cos \theta-C \sin \theta)^{2 \alpha-2 k-2} e^{\beta \theta} d \theta
\end{aligned}
$$


In this case the polynomials

$$
J_{n}^{(\alpha, \beta)}(A, B, C, D)=\frac{c_{n}}{w} D^{n}\left(w A^{n}\right), 0 \leq n<n_{w}, w=w_{0},
$$

form a finite sequence of classical orthogonal polynomials, with

$$
n_{w}=\left\lfloor\alpha-\frac{1}{2}\right\rfloor .
$$

By Theorem 1.1 and Remark 1.2 we obtain the inequalities (2.1) with

$$
\delta_{k}=\sqrt{\frac{\left(A^{2}+C^{2}\right)^{\alpha-k}}{k !(2 \alpha-2 k)_{k}(A D-B C)^{2 \alpha-2 k-1}} \int_{-\pi / 2}^{\pi / 2}(A \cos \theta-C \sin \theta)^{2 \alpha-2 k-2} e^{\beta \theta} d \theta} .
$$

They become identities for every polynomial of degree $\leq k$.

\section{Proofs of the MAIN RESUlt}

In the case when $k=0$, the proofs of Theorem 1.1 and Remark 1.2 are trivial. Otherwise, let $n_{w}$ denote the finite or infinite length of the sequence of classical orthogonal polynomials $Q_{n}$ with respect to the inner product $(\cdot, \cdot)_{w}$. Next, suppose that either $f \in L_{w_{k}}^{2, k}(a, b)$ or $f \in \mathcal{P}_{n_{w}-1}$ if $n_{w}=\infty$ or $n_{w}<\infty$, respectively. Then it follows that $f \in L_{w}^{2}(a, b)$ [22], i.e. that the distance functional is well defined. Further, in view of Parseval's identity together with the characterisation of best approximations in inner product spaces [7, we derive

$$
E_{w, k}^{2}(f)=\sum_{n=k}^{n_{w}}\left(f, \widehat{Q}_{n}\right)_{w}^{2}
$$

and

$$
\left\|D^{k} f\right\|_{w_{k}}^{2}=\sum_{n=k}^{n_{w}}\left(f, \widehat{Q}_{n}\right)_{w}^{2}\left\|D^{k} \widehat{Q}_{n}\right\|_{w_{k}}^{2}
$$

where we use the convention that the summations are over all indices $n$ such that $k \leq n<n_{w}$. Since we have

$$
\frac{E_{w, k}(f)}{\left\|D^{k} f\right\|_{w_{k}}}=\left(\sum_{n=k}^{n_{w}} \frac{\left(f, \widehat{Q}_{n}\right)_{w}^{2}}{\sum_{j=k}^{n_{w}}\left(f, \widehat{Q}_{j}\right)_{w}^{2}}\left\|D^{k} \widehat{Q}_{n}\right\|_{w_{k}}^{2}\right)^{-\frac{1}{2}}
$$

it follows that

$$
E_{w, k}(f) \leq\left(\inf _{k \leq n<n_{w}}\left\|D^{k} \widehat{Q}_{n}\right\|_{w_{k}}\right)^{-1}\left\|D^{k} f\right\|_{w_{k}} .
$$

Let us suppose for the moment that

$$
\inf _{k \leq n<n_{w}}\left\|D^{k} \widehat{Q}_{n}\right\|_{w_{k}}=\left\|D^{k} \widehat{Q}_{k}\right\|_{w_{k}} .
$$

Since $D^{k} \widehat{Q}_{k}$ is a constant distinct from zero, it follows from (1.4) that inequality (3.1) can be rewritten in the form

$$
E_{w, k}(f) \leq \frac{1}{\left|D^{k} \widehat{Q}_{k}\right|}\left\|D^{k} f\right\|_{w_{k}}
$$

whenever (3.2) holds. 
Conversely, one can apply the orthogonality of classical polynomials $D^{k} \widehat{Q}_{n}$ with respect to the inner product $(\cdot, \cdot)_{w_{k}}$ in order to get

$$
\begin{aligned}
\int_{a}^{b}\left(D^{k} f\right)(x) w_{k}(x) d x & =\sum_{n=k}^{n_{w}}\left(f, \widehat{Q}_{n}\right)_{w}\left(D^{k} \widehat{Q}_{n}, 1\right)_{w_{k}} \\
& =\left(f, \widehat{Q}_{k}\right)_{w}\left(D^{k} \widehat{Q}_{k}, 1\right)_{w_{k}}
\end{aligned}
$$

and

$$
\frac{E_{w, k}^{2}(f)}{\left[\int_{a}^{b}\left(D^{k} f\right)(x) w_{k}(x) d x\right]^{2}}=\frac{1}{\left(D^{k} \widehat{Q}_{k}, 1\right)_{w_{k}}^{2}} \sum_{n=k}^{n_{w}} \frac{\left(f, \widehat{Q}_{n}\right)_{w}^{2}}{\left(f, \widehat{Q}_{k}\right)_{w}^{2}} .
$$

Hence it is clear that

$$
\begin{aligned}
E_{w, k}(f) & \geq \frac{1}{\left|\left(D^{k} \widehat{Q}_{k}, 1\right)_{w_{k}}\right|}\left|\int_{a}^{b}\left(D^{k} f\right)(x) w_{k}(x) d x\right| \\
& =\frac{1}{\left|D^{k} \widehat{Q}_{k}\right|}\left|\int_{a}^{b}\left(D^{k} f\right)(x) w_{k}(x) d x\right| .
\end{aligned}
$$

Note that inequalities (3.1) and (3.3) become equalities for every polynomial $f$ of degree $\leq k$. Indeed, when $f$ is a polynomial of degree $<k$, then $E_{w, k}(f)=0$ and the right hand sides of (3.1) and (3.3) are also equal to zero. When $f$ is a polynomial of degree $k$, then $f=c_{k} \widehat{Q}_{k}+g$, where $c_{k} \neq 0$ is some constant and $g$ is a polynomial of degree $<k$, so that $E_{w, k}(f)=\left|c_{k}\right|$. Furthermore $D^{k} f=c_{k} D^{k} \widehat{Q}_{k}$, and hence the inequalities indeed become equalities. Therefore, the proof of Theorem 1.1 will be completed if we establish (3.2).

For this purpose, we first compute norms $\left\|D^{k} \widehat{Q}_{n}\right\|_{\widetilde{w}_{k}}$ of the derivatives of classical orthonormal polynomials $\widehat{Q}_{n}$, where $\widetilde{w}_{k}(x)=w(x) A^{k}(x), a<x<b$. First, by using (1.3), we get

$$
\begin{aligned}
(-1)^{k} D^{k}\left(\widetilde{w}_{k} D^{k} \widehat{Q}_{n}\right) & =(-1)^{k} D^{k-1}\left(\lambda_{n, k-1} \widetilde{w}_{k-1} D^{k-1} \widehat{Q}_{n}\right) \\
& =\lambda_{n, k-1}(-1)^{k} D^{k-1}\left(\widetilde{w}_{k-1} D^{k-1} \widehat{Q}_{n}\right)
\end{aligned}
$$

Hence an easy induction yields

$$
(-1)^{k} D^{k}\left(\widetilde{w}_{k} D^{k} \widehat{Q}_{n}\right)=(-1)^{k} \lambda_{n, k-1} \lambda_{n, k-2} \ldots \lambda_{n, 0} w \widehat{Q}_{n} .
$$

Consequently, integrating by parts $k$ times and using the boundary conditions for the Pearson equation, we get

$$
\begin{aligned}
\left\|D^{k} \widehat{Q}_{n}\right\|_{\widetilde{w}_{k}}^{2} & =\int_{a}^{b}\left(D^{k} \widehat{Q}_{n}\right)(x)\left[\left(D^{k} \widehat{Q}_{n}\right)(x) \widetilde{w}_{k}(x)\right] d x \\
& =(-1)^{k} \int_{a}^{b} \widehat{Q}_{n}(x) D^{k}\left[\widetilde{w}_{k}(x)\left(D^{k} \widehat{Q}_{n}\right)(x)\right] d x \\
& =(-1)^{k} \lambda_{n, k-1} \lambda_{n, k-2} \ldots \lambda_{n, 0}\left\|\widehat{Q}_{n}\right\|_{w}^{2}=(-1)^{k} \prod_{i=0}^{k-1} \lambda_{n, i},
\end{aligned}
$$


for all $n$ such that $1 \leq k \leq n<n_{w}$. Hence we obtain

$$
\gamma_{k}\left\|D^{k} \widehat{Q}_{n}\right\|_{w_{k}}^{2}=(n-k+1)_{k} \prod_{i=0}^{k-1}\left[-(n+i-1) a_{2}-b_{1}\right],
$$

where

$$
(n)_{k}=n(n+1) \cdot \ldots \cdot(n+k-1)
$$

denotes the Pochhammer symbol. It is clear that the right hand side in equation (3.4) should be positive for all $n<n_{w}$ and $k=1,2, \ldots, n$. Thus one can distinguish the following particular cases:

(i) If $a_{2} \leq 0$ and $b_{1}<0$, then $n_{w}=\infty$. By equation (3.4) the sequence $\left\|D^{k} \widehat{Q}_{n}\right\|_{w_{k}}^{2}, n=k, k+1, \ldots$, is strictly increasing, which shows the identity (3.2) and completes the proof of Theorem 1.1 for the Jacobi, Hermite and Laguerre classical weight functions.

(ii) If $a_{2}=1$ and $b_{1}<0$, then the right hand side of (3.4) is positive only if

$$
n<n_{w}=\left\lfloor\frac{1-b_{1}}{2}\right\rfloor .
$$

In this case the identity (3.4) can be rewritten in the form

$$
\gamma_{k}\left\|D^{k} \widehat{Q}_{n}\right\|_{w_{k}}^{2}=\prod_{i=0}^{k-1}\left[(n-i)\left(-n-i+1-b_{1}\right)\right]
$$

Since the quadratic polynomials $(n-i)\left(-n-i+1-b_{1}\right)$ of variable $n$ are positive and increasing on the intervals $\left(i, \frac{1-b_{1}}{2}\right)$, it follows that the sequence $\left\|D^{k} \widehat{Q}_{n}\right\|_{w_{k}}^{2}\left(k \leq n<n_{w}\right)$ is increasing. This completes the proof of (3.2), which in turn yields Theorem 1.1 for generalized Bessel, Jacobi on $[0, \infty)$ and pseudo-Jacobi classical weight functions.

Finally, the proof of Remark 1.2 is an immediate consequence of the identities

$$
\gamma_{k}\left(D^{k} \widehat{Q}_{k}\right)^{2}=\gamma_{k}\left\|D^{k} \widehat{Q}_{k}\right\|_{w_{k}}^{2}=k ! \prod_{i=0}^{k-1}\left[-(k+i-1) a_{2}-b_{1}\right],
$$

which follow from (1.4), (3.4) and the fact that $D^{k} \widehat{Q}_{k}$ is a constant independent of $x$. Moreover, the proof of Remark 1.3 is a direct corollary of the involved definitions.

\section{ACKNOWLEDGEMENT}

The authors are very grateful to the referee for suggestions that improved the paper. 


\section{REFERENCES}

1. R. P. Agarwal, G. V. Milovanović, Extremal problems, inequalities, and classical orthogonal polynomials, Applied Mathematics and Computation 128 (2002), 151-166. MR 1891017 (2003d:33012)

2. E. X. L. de Andrade, D. K. Dimitrov, A. Sri Ranga, Characterization of generalized Bessel polynomials in terms of polynomial inequalities, Journal of Mathematical Analysis and Applications 221 (1998), 538-543. MR1621746 (99b:33011)

3. G. E. Andrews, R. Askey, R. Roy, Special Functions, Encyclopedia of Mathematics and its Applications, 71, Cambridge University Press, 1999. MR1688958 (2000g:33001)

4. S. Bochner, Über Sturm-Liouvillesche Polynomsysteme, Mathematische Zeitschrift 29 (1929), 730-736. MR1545034

5. H. Chernoff, A note on an inequality involving the normal distribution, Annals of Probability 9 (1981), 533-535. MR614640 (82f:60050)

6. T. S. Chihara, An Introduction to Orthogonal Polynomials, Mathematics and its Applications, 13, Gordon and Breach, New York, 1978. MR0481884 (58:1979)

7. F. Deutsch, Best Approximation in Inner Product Spaces, Canadian Mathematical Society, Springer-Verlag, New York, 2001. MR 1823556 (2002c:41001)

8. I. S. Gradshteyn, I. M. Ryzhik, Table of Integrals, Series, and Products, Academic Press, New York-London, 1965. MR0197789 (33:5952)

9. W. Hahn, Über die Jacobischen Polynome und zwei verwandte Polynomklassen, Mathematische Zeitschrift 39 (1935), 634-638. MR1545524

10. M. E. H. Ismail, Classical and Quantum Orthogonal Polynomials in One Variable, Encyclopedia of Mathematics and its Applications, 98, Cambridge University Press, 2005. MR2191786 (2007f:33001)

11. R. Koekoek, R. F. Swarttouw, The Askey-scheme of hypergeometric orthogonal polynomials and its q-analogue, Report 98-17 of the Faculty of Technical Mathematics and Informatics, Delft University of Technology, 1998.

12. W. Koepf, M. Masjed-Jamei, A generic polynomial solution for the differential equation of hypergeometric type and six sequences of orthogonal polynomials related to it, Integral Transforms and Special Functions 17 (2006), 559-576. MR2246501(2008b:33021)

13. H. L. Krall, On derivatives of orthogonal polynomials, Bulletin of the American Mathematical Society 42 (1936), 423-428. MR.1563314

14. H. L. Krall, On higher derivatives of orthogonal polynomials, Bulletin of the American Mathematical Society 42 (1936), 867-870. MR.1563455

15. H. L. Krall, On derivatives of orthogonal polynomials. II, Bulletin of the American Mathematical Society 47 (1941), 261-264. MR0003854 (2:282e)

16. K. H. Kwon, L. L. Littlejohn, B. H. Yoo, New characterizations of classical orthogonal polynomials, Indagationes Mathematicae 7 (1996), 199-213. MR1621324 (99g:33026)

17. P. A. Lesky, Endliche und unendliche Systeme von kontinuierlichen klassischen Orthogonalpolynomen, Zeitschrift für Angewandte Mathematik und Mechanik 76 (1996), 181-184. MR.1382858 (97e:33009)

18. M. Masjed-Jamei, Classical orthogonal polynomials with weight function $\left((a x+b)^{2}+\right.$ $\left.(c x+d)^{2}\right)^{-p} \exp (q \operatorname{Arctg}((a x+b) /(c x+d))), x \in(-\infty, \infty)$ and a generalization of $T$ and $F$ distributions, Integral Transforms and Special Functions 15 (2004), 137-153. MR2053407 (2005b:33011)

19. A. F. Nikiforov, V. B. Uvarov, Special Functions of Mathematical Physics, Birkhäuser Verlag, Basel, 1988. MR.922041 (89h:33001)

20. V. Romanovski, Sur quelques classes nouvelles de polynômes orthogonaux, Comptes Rendus hebdomadaires des séances de l'Académie des sciences 188 (1929), 1023-1025.

21. V. D. Stepanov, An extremal property of Chebyshev polynomials, Proceedings of the Steklov Institute of Mathematics 248 (2005), 230-242. MR2165931 (2006h:41006)

22. V. D. Stepanov, An extremal property of Jacobi polynomials in two-sided Chernoff-type inequalities for higher order derivatives, Proceedings of the American Mathematical Society 136 (2008), 1589-1597. MR2373588 (2009i:26024)

23. P. K. Suetin, Classical Orthogonal Polynomials (in Russian), Nauka, Moscow, 1979. MR.548727 (80h:33001) 
24. G. Szegö, Orthogonal Polynomials, American Mathematical Society Colloquium Publications, Volume XXIII, Amer. Math. Soc., New York, 1939. MR0000077 (1:14b)

25. M. S. Webster, Orthogonal polynomials with orthogonal derivatives, Bulletin of the American Mathematical Society 44 (1938), 880-888. MR.1563895

Department of Mathematics and Computer Science, The John Paul II Catholic University of Lublin, ul. Konstantynów 1h, 20-708 Lublin, Poland

E-mail address: rsmax@kul.lublin.pl

Department of Mathematics and Computer Science, The John Paul II Catholic University of Lublin, Ul. Konstantynów 1h, 20-708 Lublin, Poland

E-mail address: rootus@kul.lublin.pl 